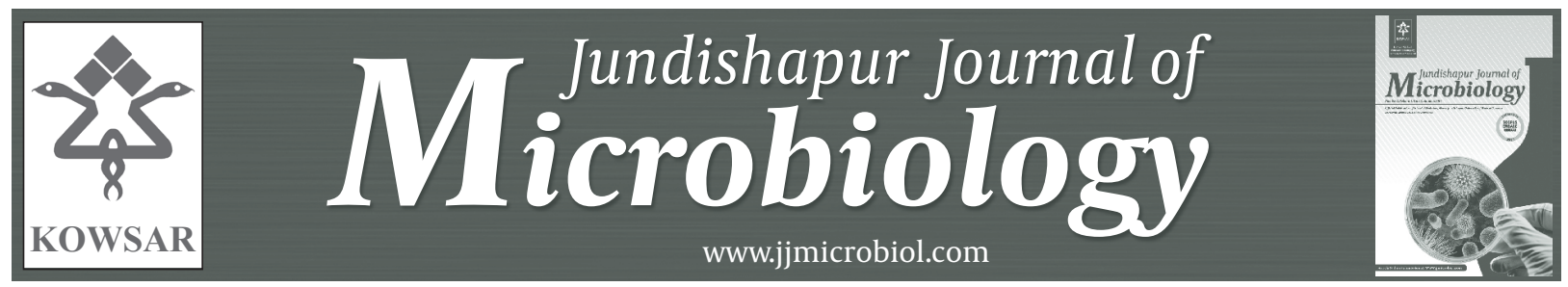

\title{
Immunostimulatory Effects of Aqueous Extract of Heracleum persicum Desf. on Mouse Peritoneal Macrophages
}

\author{
Alireza Naeini ${ }^{1,{ }^{*}}$, Hojjatollah Shokri ${ }^{2}$, Ali Reza Khosravi ${ }^{3}$ \\ ${ }^{1}$ Traditional Medicine Clinical Trail Research Center, Shahed University, Tehran, IR Iran \\ ${ }^{2}$ Faculty of Veterinary Medicine, Amol University of Special Modern Technologies, Amol, IR Iran \\ ${ }^{3}$ Mycology Research Center, Faculty of Veterinary Medicine, University of Tehran, Tehran, IR Iran \\ *Corresponding author: Alireza Naeini, Department of Mycology, Faculty of Medicine and Traditional Medicine Clinical Trail Research Center, Shahed Uni- \\ versity, Keshavarz Blvd, P.O. Box: 14155-7435, Tehran, IR Iran. Tel: +98-2188964792, Fax: +98-2188966310, E-mail: aemamh@yahoo.com, alinaeini@hotmail. \\ com.
}

\section{A B S T R A C T}

Background: The immune system provides protection against infectious diseases that are caused by various microorganisms, in particular pathogenic fungi. Utilization of herbal immunostimulants is one solution to improve the immunity of humans and to decrease their susceptibility to infectious diseases.

Objectives: The current study aimed to investigate the immunostimulatory effects of the aqueous extract of Heracleum persicum on mouse peritoneal macrophages.

Materials and Methods: The present in vitro study investigated the effect of the aqueous extract of $H$.perscium on the viability of macrophages and nitric oxide (NO) production using microculture tetrazolium (MTT) assay and Griess method, respectively. The effects on fungicidal activity and reactive oxygen species (ROS) production of stimulated peritoneal macrophages were also studied using killing method and nitroblue tetrazolium (NBT) assay, respectively.

Results: The aqueous extract of H. persicum (Hp-W) at concentration of $10 \mathrm{mg} / \mathrm{mL}$ resulted in a significant increase in NO production (8.17 $\mathrm{nmol}$ ) by macrophages $(\mathrm{P}<0.05)$. Moreover, $H$. persicum had a stimulatory effect on the level of $\mathrm{ROS}(\mathrm{P}<0.05)$ and a strong candidacidal activity in macrophages treated with $20 \mathrm{mg} / \mathrm{mL}$ of the extract $(\mathrm{P}<0.05)$.

Conclusions: The aqueous extract of $H$. persicum showed a significant immunostimulatory activity on macrophages. To clarify the exact mechanisms of this activity, more studies should be done with purified immunostimulatory components of $H$. persicum in future.

Keywords: Immunostimulatory activity; Heracleum persicum; MTT assay; NBT assay; Killing, Candida albicans

Copyright @ (2013, Ahvaz Jundishapur University of Medical Sciences; Published by Kowsar Corp.

Article type: Research Article; Received: 01 May 2012; Revised: 17 Jul 2012; Accepted: 08 Aug 2012; Epub: 01 Jun 2013; Ppub: Jun 2013

Implication for health policy/practice/research/medical education:

The results demonstrated that natural immunostimulants, in particular Heracleum persicum, are attractive alternatives exerting synergistic effects on host immunity as well as against pathogenic Candida species.

-Please cite this paper as:

Naeini A, Shokri H, Khosravi AR. Immunostimulatory Effects of Aqueous Extract of Heracleum persicum Desf. on Mouse Peritoneal Macrophages. Jundishapur J Microbiol. 2013;6(4):e5373. DOI: 10.5812/jjm.5373.

Copyright @ 2013, Ahvaz Jundishapur University of Medical Sciences; Published by Kowsar Corp.

This is an Open Access article distributed under the terms of the Creative Commons Attribution License (http://creativecommons.org/licenses/by/3.0), which permits unrestricted use, distribution, and reproduction in any medium, provided the original work is properly cited. 


\section{Background}

Innate immunity has a key role in preventing the host against opportunistic infections. Among various immune cells, macrophages are one of the research foci of the immunology community. Macrophages play a significant role against many opportunistic microbs especially Candida species (1). Candida albicans is an important commensal microflora on digestive tracts and mucosal barriers (2). The host immune system is the major factor balancing the transition from commensalism to pathogenicity of C.albicans (3).

Candida infections occur predominantly in patients who suffer some kinds of systemic or local immunosuppression such as persons with diabetes, patients infected with HIV, patients receiving corticosteroid or cytotoxic chemotherapy particularly for hematologic malignancies, persons exposed to prolonged antibiotic treatment and recipients of organ or stem cell transplantation (4). Reactive oxygen species (ROS) and nitric oxide (NO) are also the main mechanisms by macrophages for killing these fungal agents (5).

One of the most promising alternatives to classical antibiotic treatment is the use of immunomodulators to enhance the host defense response (6). There are several immunomodulators with botanical origins such as mushrooms, algae, lichens and higher plants. In this regard, some herbs with immunomodulartory activities including Viscum album (V. album), Withania somnifera and Allium sativum have been reported by different investigators (6-8). The predominant components including polysaccharides, lectins, proteins and peptides are known to stimulate the immune system (6, 9). Davis and Kuttan (7) showed that treatment with $20 \mathrm{mg}$ of $W$. somnifera root extract resulted in an enhancement in phagocytic activity of peritoneal macrophages. In a study by Choi et al. (10), the methanolic extract of Caloplacaragalis (CR-ME) increased the production of tumor necrosis factor- $\alpha$ (TNF$\alpha$ ) and NO by peritoneal macrophages. However, CR-ME had a little effect on the levels of ROS.

H. persicum, commonly known as Golpar in Persian, is a flowering plant in the Apiaceae family that grows wild in humid alpine regions of Iran $(11,12)$. This aromatic plant is used as a flavoring ingredient in most of Iranian food products. Based on Iranian traditional medicine, it is used to relieve flatulence and stomach aches. It is also used to disinfect the stomach, antioxidants, and cure a poor appetite $(13,14)$. Preliminary phytochemical analysis of $H$. persicum extract showed the presence of alkaloids, terpenoids, triterpenes and steroids (15).

\section{Objectives}

The current study was undertaken to estimate the in vitro stimulatory effects of aqueous extract of $\mathrm{H}$. persicum on viability, NO and ROS productions of peritoneal mac- rophages and candidacidal activity.

\section{Materials and Methods}

\subsection{Plant Collection and Identification}

The aerial parts of $H$. persicum were harvested from Khorasan province, northeast of Iran,in 2007. Botanical identification was performed at the Herbarium of Pharmacognosy Department, School of Pharmacy, Shaheed Beheshti University of Medical sciences, Iran. The voucher botanic specimen was 1312 .

\subsection{Preparation of Extraction}

The plant seeds were ground into fine powder. For aqueous extract preparation, $100 \mathrm{~g}$ of plant powder was mixed with $400 \mathrm{~mL}$ of water, boiled for $10 \mathrm{~min}$ and filtered by Whatman paper (No.1). The resulting solution was frozen and lyophilized for $96 \mathrm{~h}$ at $-50^{\circ} \mathrm{C}$ and 0.04 mbar (Snijder scientific Ltd, Holland). The residue was coded with letter (Hp-W).

\subsection{Candida albicans Strain}

Candida albicans (ATCC 10231) was cultured on Sabouraud glucose agar (Merck Co., Darmstadt, Germany) at $35^{\circ} \mathrm{C}$ for 3 days, harvested and kept at $4^{\circ} \mathrm{C}$ until used.

\subsection{Animals and Peritoneal Macrophages Prepa- ration}

Male Balb/c mice (6 to 8 weeks of age, weighting 18-25 g) were purchased from the Animal Breeding Laboratory of the Faculty of Medicine, Shahed University, Tehran, Iran. All animals were housed and handled according to institutionally recommended guidelines.

The animals were sacrificed and peritoneal exudates cells were harvested by lavage using $5 \mathrm{~mL}$ of cold PBS (5 $\mathrm{mg} / \mathrm{mL}, \mathrm{pH} 7.2$ ) and poured in sterile plastic tubes. Cells were pooled, resuspended in RPMI1640 supplemented with 5\% FBS (GIBCO, Grand Island, NY, USA) and cultured in 96-well flat-bottom microtiter plates at a final concentration of $4 \times 10^{5}$ cells per well. After $2 \mathrm{~h}$, the debris and non-adherent cells were removed from the wells. Then, the monolayer macrophages were reincubated at $37^{\circ} \mathrm{C}$ for $20 \mathrm{~h}$ along with different concentrations of extract (5, 10 and $20 \mathrm{mg} / \mathrm{mL}$ ).

\subsection{Macrophages Viability Assay}

MTT \{3- (4, 5-dimethylthiaozle-2-yl) -2, 5 - diphenyle - 
tetra zolium bromide\} powder (Merck Co., Darmstadt, Germany) was dissolved in PBS (5 mg/mL, pH 7.4), filtered and stored at $-20^{\circ} \mathrm{C}$ until used. The MTT assay was performed in the 96-well plates (16). Briefly, the wells were washed three times with complete medium, then $180-\mu 1$ aliquots of medium and 20- $\mu$ l aliquots of MTT solution (5 $\mathrm{mg} / \mathrm{mL}$ of PBS) were added to each well at the established time. After $2 \mathrm{~h}$ of incubation at $37^{\circ} \mathrm{C}$ and 5\% CO2 for exponentially growing cells and 15 min for steady-state confluent cells, the media were removed and formazan crystals were solubilized with $175 \mu$ l of DMSO. The plates were then read on a Microplate reader Model 450 (Bio-Rad Laboratories, Hercules, CA, USA) at 540-nm wave length.

\subsection{Extracellular NO Production}

NO released into the supernatants of mouse macrophages was determined by the standard Griess reaction by adding $50 \mu \mathrm{l}$ of test solution to 96 -well flat-bottomed plates containing $50 \mu \mathrm{l}$ of Griess reagent [1\% sulfanilamide/0.1\% N-(1-naphthyl) ethylenediamine dihydrochloride $2.5 \% \mathrm{H} 3 \mathrm{PO} 4$ ]. The samples were assayed in quadruplicate. After $15 \mathrm{~min}$ at room temperature, the absorbance of each well was measured in a Multiskan MS microplate reader (Labsystems Oy, Helsink, Finland) at $540 \mathrm{~nm}$ and the nitrite concentration was determined from a standard curve of sodium nitrite (17).

\subsection{Intracellular ROS Production}

Determination of intracellular ROS production by macrophages was determined using NBT assay by Gentle and Thompson method (18). Briefly, peritoneal macrophages were seeded at a density of $1 \times 10^{5}$ cells per well and treated with aqueous extract of $H$. persicum (Hp-W, $20 \mathrm{mg} / \mathrm{mL}$ ) with or without N-formyl-methionyl-leucyl-phenylalanine plus lipopolysaccharide (f MLP+LPS) stimulator for $20 \mathrm{~h}$ in 96 -well flat-bottom microtiter plate. Then, $50 \%$ of RPMI and $50 \%$ of NBT sterile solution $(0.1 \%)$ were added to each well and incubated at $37^{\circ} \mathrm{C}$ for $1 \mathrm{~h}$. The supernatant was removed, and $50 \mathrm{~mL}$ of pyridine was added, and absorbances were read using ELISA reader at $540 \mathrm{~nm}$. Binding of macrophage receptors with f MLP and LPS resulted in hydrolysis of phosphatidylinositol diphosphate by a specific phospholipase $\mathrm{C}$, subsequently leading to a rise in inositol trisphosphate, secretion of lysosomal enzymes, NADPH oxidase and ROS production (19).

\subsection{Candidacidal Activity of Macrophages}

Candida albicansATCC 10231 was used in the fungicidal assay as a target microorganism. Macrophages $\left(1 \times 10^{6}\right.$ cell $/ \mathrm{mL}$ ) were pre-incubated with $\mathrm{Hp}-\mathrm{W}$ for $3 \mathrm{~h}$, and then the supernatant was replaced with fresh medium. Macro- phages and C. albicans were mixed at 1:1 ratio and incubated under constant rotation at $39.5^{\circ} \mathrm{C}$ and $5 \% \mathrm{CO} 2$. After $1 \mathrm{~h}$, $50 \mathrm{~mL}$ of the suspension were added to $4.95 \mathrm{~mL}$ of chilled water and thoroughly mixed. Subsequently, $50 \mathrm{~mL}$ from this suspension was distributed on a Petri dish containing Sabouraud glucose agar (Merck Co., Darmstadt, Germany) and incubated at $37^{\circ} \mathrm{C}$ for $48 \mathrm{~h}$. The colonies were counted and data were illustrated as follow (3):

Fungicidal activity $=[1$ - CFU experimental culture $/ \mathrm{CFU}$ untreated $] \times 100$

\subsection{Statistical Analysis}

Data were analyzed using a one-way analysis of variances (ANOVA) and presented as Mean + SEM. The P values < 0.05 were considered as significant differences (SPSS version 10).

\section{Results}

\subsection{Macrophages Viability}

Regarding the effect of the aqueous extract of $H$. persicum (Hp-W) on viability of macrophages, there were no significant differences between Hp-W and control group at the applied doses ( Figure 1).

Figure 1. The Viability of the Peritoneal Macrophages Treated with the Aqueous Extract of $H$. persicum (Hp-W) for $16 \mathrm{~h}$ (Mean \pm SEM).

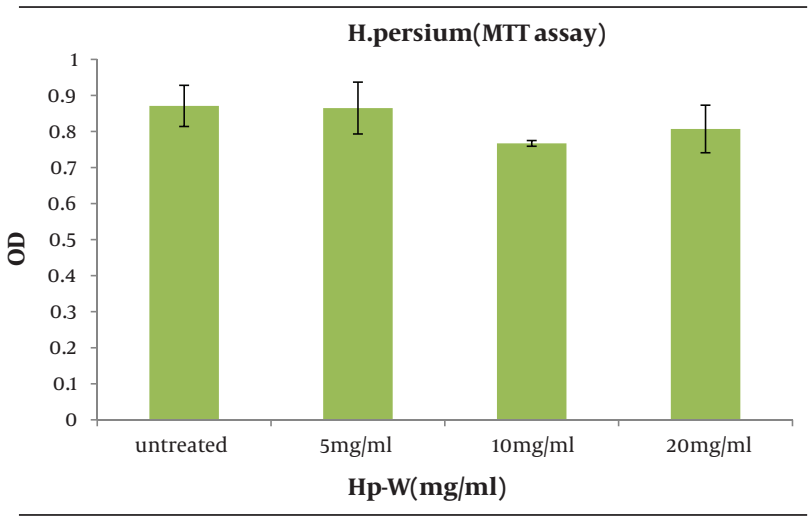

Significant differences were not observed among different tested groups $(P>0.05)$.

\subsection{NO Production}

As shown in Figure 2 , the aqueous extract of $H$. persicum (Hp-W) at the concentration of $10 \mathrm{mg} / \mathrm{mL}$ induced a significant increase in NO production when compared to control group $(\mathrm{P}<0.05)$. The concentrations of NO production in control group and macrophages treated with $\mathrm{Hp}-\mathrm{W}(10 \mathrm{mg} / \mathrm{mL})$ were 2.89 and $8.17 \mathrm{nmol}$, respectively. 
Figure 2. NO Production of Peritoneal Macrophages Stimulated With the Aqueous Extract of $H$. persicum (Hp-W) for $16 \mathrm{~h}$ (Mean \pm SEM).

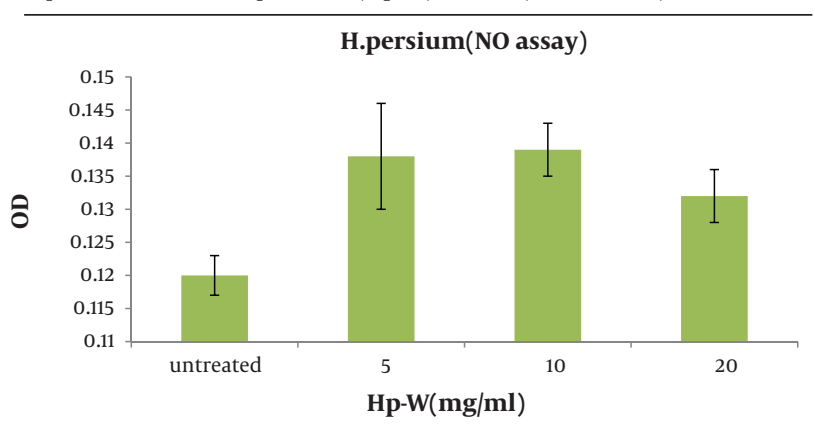

* Significant difference was observed between Hp-W (at concentration of $10 \mathrm{mg} / \mathrm{mL})$ and control $(\mathrm{P}<0.05)$.

\subsection{ROS Production}

The effect of Hp-W on ROS production was given in Figure 3 . Increasing ROS production was found significant by $\mathrm{Hp}-\mathrm{W}$ at the concentration of $20 \mathrm{mg} / \mathrm{mL}(\mathrm{P}<0.05)$. In addition, combination of Hp-W at $20 \mathrm{mg} / \mathrm{mL}$ with $\mathrm{f}$ MLP + LPS significantly stimulated ROS production compared to the control group $(\mathrm{P}<0.05)$, but there was no statistically significant difference between Hp-W and f MLP + LPS, which means that $\mathrm{fMLP}$ and LPS cannot increase ROS production.

Figure 3. ROS Production of Peritoneal Macrophages Stimulated With the Aqueous Extract of H. persicum (Hp-W) and Hp-W + Stimulators (LPS+f MLP) for $16 \mathrm{~h}($ Mean \pm SEM).

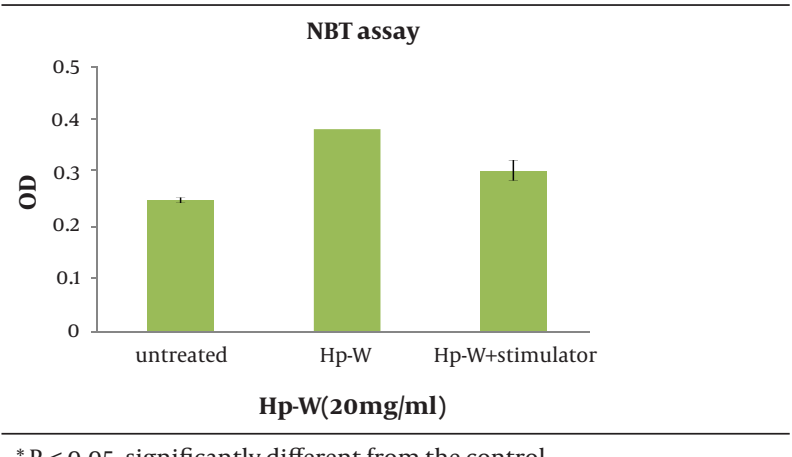

${ }^{*} \mathrm{P}<0.05$, significantly different from the control.

\subsection{Killing of C. albicans by Macrophages}

In order to evaluate the possible effect of the aqueous extract on the fungicidal activity of macrophages against C. albicans, fungicidal activity test was performed. As illustrated in Table 1, candidacidal activity of macrophages augmented significantly at the presence of $\mathrm{Hp}-\mathrm{W}$ extract. According to Figure 4 , killing of the macrophages (51.9\%) were significantly stimulated with Hp-W extract (lower number of Candida colonies were obtained) at the concentration of $20 \mathrm{mg} / \mathrm{mL}$ when compared to control $\operatorname{group}(\mathrm{P}<0.05)$.
Table 1. Fungicidal Activity of Peritoneal Macrophages Treated With the Aqueous Extract of H.persicum (Hp-W) in Different Concentrations and Challenged with C. albicans.

\begin{tabular}{llll}
\hline $\begin{array}{l}\text { Dose, } \\
\text { mL }\end{array}$ & $\begin{array}{l}\text { N u m b e r } \\
\text { of Colony, } \\
\text { Mean } \pm \text { SEM }\end{array}$ & $\begin{array}{l}\text { Macrophage } \\
\text { Fungicidal } \\
\text { Activity, \% }\end{array}$ & Pvalue \\
\hline $\mathbf{2 0}$ & $44.6 \pm 1.2$ & 51.9 & 0.036 \\
$\mathbf{1 0}$ & $78.3 \pm 9.5$ & 15.6 & $\mathrm{NS}^{\mathrm{a}}$ \\
\hline Control & $92.8 \pm 11.8$ & 0 & $\mathrm{NS}^{\mathrm{a}}$ \\
\hline${ }^{\mathrm{a}}{ }_{\mathrm{NS}=\text { Not Significant }}$ & &
\end{tabular}

${ }^{a}$ NS=Not Significant

Figure 4. Number of Colonies After Co-Culture of the Aqueous Extract of H. persicum (Hp-W) Treated and Untreated (Control) Macrophages in Order to Evaluate the Fungicidal Activity Against C. albicans.

H.persium(Killing)

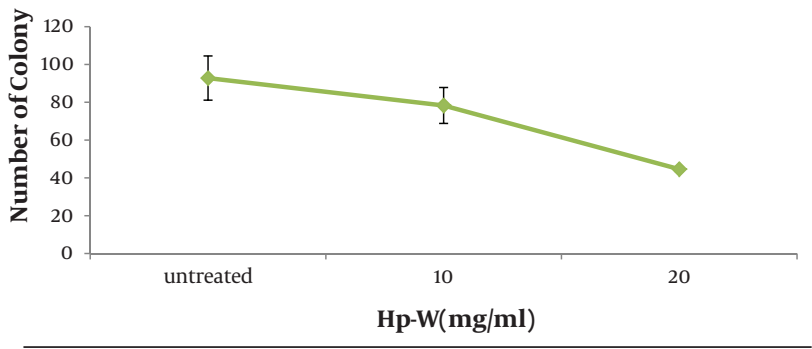

${ }^{*} \mathrm{P}<0.05$, significantly different from the control.

\section{Discussion}

The study of host resistance against systemic fungal infections has received considerable attention in the past decade (16). Until that time, most immunological investigations of these organisms concerned with the isolation and characterization of antigens for use in vaccines, diagnoses and epidemiological studies. The lack of information about the role of local defenses, antibody production and cell-mediated reactions has prompted investigations into host response to various fungi. Recent studies have pointed out the importance of innate immunity in fungal infections $(17,20)$.

Macrophages have an important role in the initial responses to infection before action of humoral and cellular immunity $(3,21)$. The function of macrophages includes phagocytosis, antigen processing and presentation, cytokine secretion and antibody dependent cell-mediated cytotoxicity $(4,5)$. It is approved that macrophage phagocytosis is a key to prevent Candida species invasion. These cells can damage and kill different shapes of C. albicans (yeast and hyphae) by oxidative and non-oxidative productions. Based on the literature review, there are few studies on the potential effects of herbal extracts on innate immunity $(22,23)$.

The present study evaluated the effect of native herbal extract of $H$. persicum on different activities of macro- 
phages. The results showed no significant difference between the effects of aqueous extract (Hp-W) and control on viability of macrophages at the applied concentrations. The aqueous extract of $H$. persicum (Hp-W) at concentration of $10 \mathrm{mg} / \mathrm{mL}$ induced a significant increase in NO production compared to the control group. Application of $\mathrm{Hp}-\mathrm{W}$ extract at $20 \mathrm{mg} / \mathrm{mL}$ significantly increased ROS production as well. These activities could be due to the presence of flavonoids and coumarins, which can augment the macrophage responses (24). As far as we know, little information has been reported on immunostimulatory effect of $H$. persicum, although there are similar works on various herbs. In a study conducted by Sharififar et al. (25), H. persicumshowed a stimulatory effect on both humoral and cellular immune functions in mice.

The $H$. persicum extract elicited a significant increase $(\mathrm{P}<0.05)$ in the delayed type hypersensitivity response at doses of 100 and $200 \mathrm{mg} / \mathrm{kg}$. There are some studies which have confirmed the immunostimulatory effects of the two other species of H. maximum and H. nepalense (26, 27). The extract of $H$. maximum stimulates the production of IL- 6 which its production is a well-established and reliable marker of macrophage activation (27). It has been shown that the methanolic extract of $H$. nepalense, at a dose of $1000 \mathrm{mg} / \mathrm{kg}$, results in a four-fold increase in haemaglutinin titer when compared to control group (26).

More specifically, C. albicans blastoconida have shown to be susceptible to the oxygen-dependent killing mechanisms of mononuclear phagocytic cells. The candidacidal activity of mononuclear phagocytic cells has been associated with the production of superoxide anion, one of the products of reactive oxygen species (ROS) metabolism which is essential for macrophages "oxidative killing" (5). Interestingly, with respect to ROS production, there was no significant difference between Hp-W and combination of stimulators with Hp-W. The exact mechanisms underlying this event remains unclear, but it seems that oral exposure of Hp-W at concentration of $20 \mathrm{mg} / \mathrm{mL}$ will be able to induce ROS production with the same strength that mitogens such as f MLP and LPS do, through different signal transduction pathways (28). Therefore, as f MLP and LPSare known as strong macrophage stimulators, $\mathrm{Hp}-\mathrm{W}$ is also a suitable activator and can be included in macrophage stimulator agents.

The present study demonstrated that Hp-W at the concentration of $20 \mathrm{mg} / \mathrm{mL}$ significantly increased candidacidal activity of macrophage compared to control group. Naeini et al. (29) showed anti-C.albicans activity of essential oil of H. persicum. Static and lethal effects of the above oil against C. albicans were $1.1 \mathrm{mg} / \mathrm{mL}$, representing moderate efficacy against this Candida species. The valuable effects of $H$. persicumhave been reported. In a study by Souri et al. (30), the antioxidant activity of some furanocoumarins isolated from $H$. persicum was demonstrated. According to the results, antioxidant activity of crude ethyl acetate extract was stronger than that of isolated from single component. Sayyah (15) exhibited the anticonvulsant activity of acetonic extract of the seeds of $H$. persicum against pentylenetetrazole (PTZ) and maximal electroshock (MES)-induced seizures in mice. The extract showed a dose-dependent protective effect in both seizure models. The observed pharmacological effects could be due to alkaloids, terpenoids, and triterpenes present in the plant (15).

Finally, $H$. persicum can be used to enhance innate immune functions, in particular macrophage activity. Since $H$. persicum plant has been used as food additive from ancient time up to now, so the Hp-W can be administrated orally without any known side effects. The role of different components of $H$. persicum extract on the macrophage function is not fully understood. So, further studies on the effects of the components on immune cells are required and this study should be continued to establish the extract for using these components in the exact patients.

\section{Acknowledgements}

The authors would like to thank Immunology Department of Shahed University for providing the immunologic tests. Also, they are grateful to Mr. H. Smaeil-Zade Nami and Mr. D. Jamali for their kind helps.

\section{Financial Disclosure}

None Declared.

\section{Funding/Support}

None Declared.

\section{Authors' Contribution}

None Declared.

\section{References}

1. Ebtekar M, Yaraee R, Ahmadiani A, Sabahi F. Kinetics of nitric oxide production and MTT reduction by HSV-1 infected macrophages. Iran J Med Sci. 2006;31(1):9-13.

2. Khan ZU, Chandy R, Metwali KE. Candida albicans strain carriage in patients and nursing staff of an intensive care unit: a study of morphotypes and resistotypes. Mycoses. 2003;46(11-12):479-86.

3. Loyola W, Gaziri DA, Gaziri LC, Felipe I. Concanavalin A enhances phagocytosis and killing of Candida albicans by mice peritoneal neutrophils and macrophages. FEMS Immunol Med Microbiol. 2002;33(3):201-8.

4. Tavanti A, Campa D, Bertozzi A, Pardini G, Naglik JR, Barale R, et al. Candida albicans isolates with different genomic backgrounds display a differential response to macrophage infection. Microbes Infect. 2006;8(3):791-800.

5. Vazquez-Torres A, Balish E. Macrophages in resistance to candidiasis. Microbiol Mol Biol Rev. 1997;61(2):170-92.

6. Ghazanfari T, Hassan ZM, Ebrahimi M. Immunomodulatory activity of a protein isolated from garlic extract on delayed type hypersensitivity. Int Immunopharmacol. 2002;2(11):1541-9.

7. Davis L, Kuttan G. Immunomodulatory activity of Withania somnifera.J Ethnopharmacol. 2000;71(1-2):193-200.

8. Thejass P, Kuttan G. Immunomodulatory activity of Sulforaphane, a naturally occurring isothiocyanate from broccoli (Bras- 
sica oleracea). Phytomedicine. 2007;14(7-8):538-45.

9. Hajto T, Hostanska K, Gabius HJ. Modulatory potency of the betagalactoside-specific lectin from mistletoe extract (Iscador) on the host defense system in vivo in rabbits and patients. Cancer Res. 1989;49(17):4803-8.

10. Choi HS, Yim JH, Lee HK, Pyo S. Immunomodulatory effects of polar lichens on the function of macrophages in vitro. Mar Biotechnol (NY). 2009;11(1):90-8.

11. Mirheidar H. Plant education. Farhang-e-Slami Press. 1996:403 p.

12. Sefidkon F, Dabiri M, Mohammad N. Analysis of the oil of Heracleum persicum L.(Leaves and Flowers). J Essent Oil Res. 2002;14(4):295-297.

13. Jorjani SE. Al-Aghraz al-tibbia val Mobahess al-alaiia. 1st ed.: Iran: Tehran Press; 2005.

14. Salehi Surmaghi H. Medicinal plants and phytotherapy. 2006.

15. Sayyah M, Moaied S, Kamalinejad M. Anticonvulsant activity of Heracleum persicum seed.J Ethnopharmacol. 2005;98(1-2):209-11.

16. Klepser ME. Antifungal resistance among Candida species. Pharmacotherapy. 2001;21(8 Pt 2):124S-132S.

17. Rao YK, Fang SH, Tzeng YM. Inhibitory effects of the flavonoids isolated from Waltheria indica on the production of NO, TNFalpha and IL-12 in activated macrophages. Biol Pharm Bull. 2005;28(5):912-5.

18. Gentle TA, Thompson RA. clinical Immunology, A practical Approach. Edited by HC Gool. 1990.

19. Baggiolini M, Boulay F, Badwey JA, Curnutte JT. Activation of neutrophil leukocytes: chemoattractant receptors and respiratory burst. FASEB J. 1993;7(11):10 04-10.

20. Eggimann P, Garbino J, Pittet D. Management of Candida species infections in critically ill patients. Lancet Infect Dis. 2003;3(12):77285.
21. Anaissie EJ, McGinnis MR, Pfaller MA. Clinical mycology with CDROM. 2009.

22. Mediratta PK, Sharma KK, Singh S. Evaluation of immunomodulatory potential of Ocimum sanctum seed oil and its possible mechanism of action. JEthnopharmacol. 2002;80(1):15-20.

23. Salem ML, Hossain MS. Protective effect of black seed oil from Nigella sativa against murine cytomegalovirus infection. Int J Immunopharmacol. 2000;22(9):729-40.

24. Makare N, Bodhankar S, Rangari V. Immunomodulatory activity of alcoholic extract of Mangifera indica L. in mice. JEthnopharmacol. 2001;78(2-3):133-7.

25. Sharififar F, Pournourmohammadi S, Rastegarianzadeh R, Ranjbaran $\mathrm{O}$, Purhemmaty A. Immunomodulatory activity of aqueous extract of Heracleum persicum Desf. In mice. Iran J Pharm Res. 2010;8(4):287-292.

26. Dash S, Nath LK, Bhise S, Kar P, Bhattacharya S. Stimulation of immune function activity by the alcoholic root extract of Heracleum nepalense D. Don. Indian J Pharm. 2006;38(5):336.

27. Webster D, Taschereau P, Lee TD, Jurgens T. Immunostimulant properties of Heracleum maximum Bartr. J Ethnopharmacol 2006;106(3):360-3.

28. MacEwan DJ. TNF receptor subtype signalling: differences and cellular consequences. Cell Signal. 2002;14(6):477-92.

29. Naeini A, Khosravi AR, Chitsaz M, Shokri H, Kamlnejad M. AntiCandida albicans activity of some Iranian plants used in traditional medicine. J Med Mycol. 2009;19(3):168-172.

30. Effat Souri, Hassan Farsam, Parisa Sarkheil, Fahimeh Ebadi. Antioxidant Activity of Some Furanocoumarins Isolated from Heracleum persicum. Pharmaceut Biol (Formerly International Journal of Pharmacognosy). 2004;42(6):396-399. 